\title{
Estratégias de Coping em educadores de infância portugueses
}

\author{
Coping em educadores
}

\author{
Rosa Maria Silva Gomes \\ Anabela Maria Sousa Pereira
}

\section{Resumo}

O presente estudo teve como objectivo identificar os mecanismos de coping (estratégias para lidar com situações difíceis) utilizados por Educadores de Infância quando se encontram em situações de stresse, bem como estudar as características do respectivo instrumento de avaliação na versão Portuguesa. A amostra é constituída por 247 Educadores de Infância (diplomados e em situação de estágio pedagógico) com idades compreendidas entre os 20 e os 57 anos de idade. Da Bateria Differential Stress Inventory (DSI), de Lefèvre e Kubinger (2004) foi utilizado o questionário Estratégias de Coping (EC) tipo Likert, adaptada para a população portuguesa por Gomes, Pereira e Gil (2006), revelando boas características psicométricas. Os principais resultados mostram que perante situações indutoras de stresse os Educadores de Infância utilizam mais as estratégias de coping focadas na emoção, do que as estratégias de coping focadas no problema. São referidas ainda algumas implicações deste estudo para a formação destes profissionais.

Palavras-chave: Coping, Stresse, Educação.

\section{Coping Strategies in portuguese kindergarten teachers}

\begin{abstract}
The aim of this study is to identify the mechanisms of coping (strategies to deal with difficult situations) used by kindergarten Teachers when they face situations of stress, as well as to study respective characteristics of the used evaluation instrument in a Portuguese version. The sample is constituted by 247 kindergarten teachers (graduated and training students) with ages between 20 and 57 years old. The Strategies of Coping (EC) likert type scale from the Differential Stress Inventory (DSI), of Lefèvre \& Kubinger (2004) was adapted for the Portuguese population by Gomes, Pereira \& Gil (2006), which revealed good psychometric characteristics. The main results show that kindergarten Teachers, when experiencing stress inducing situations, frequently appeal to coping strategies with focus in emotion, than based in the problem solving situation. Will be discussed further implications of this research to the professional training.

Keywords: Coping, Stress, Education.
\end{abstract}

\section{Estrategias de Coping en profesores infantiles portugueses}

\begin{abstract}
Resumen
Este estudio tuvo como objetivo identificar los mecanismos de coping (estrategias para lidiar con situaciones difíciles) utilizados por profesores infantiles cuando están en situación de stress, así como también estudiar las características del respectivo instrumento de evaluación en la versión Portuguesa. La muestra es constituida por 247 profesores infantiles (diplomados y en situación de práctica pedagógica) con edades entre los 20 y los 57 años de edad. De la Bateria Differential Stress Inventory (DSI), de Lefèvre y Kubinger (2004) fue utilizado el cuestionario Estratégias de Coping (EC) tipo Likert, adaptada para la población portuguesa por Gomes, Pereira y Gil (2006), revelando buenas características psicométricas. Los resultados principales muestran que frente a situaciones incitadoras de stress los profesores infantiles utilizan más las estrategias de coping focalizadas en la emoción que las estrategias de coping focalizadas en el problema. Todavía, son mencionadas algunas implicaciones de este estudio para la formación de estos profesionales.

Palabras clave: Coping, Stress, Educación.
\end{abstract}




\section{Introdução}

\section{O Stresse Ocupacional}

Ser Educador é, actualmente, uma profissão que apresenta constantes e complexos desafios e que invoca uma forte especificidade docente. No desempenho das suas funções, é exigido ao profissional em Educação de Infância, tomadas de decisão e subsequentes interacções em redes sociais, que não se circunscrevem à criança e ao grupo de crianças, mas que apontam a assunção de papéis sociais múltiplos, dos quais colocamos em destaque 0 de conceptualizador curricular, interventor/contextualizador, observador, avaliador, pesquisador, promotor da inteligibilidade da infância, epistemologicamente sustentada no seio da comunidade educativa (Abrantes, 2006; Guerra, 2002; Leite, 2002). Tais exigências profissionais podem provocar desajustes e tensões entre as situações profissionais, a capacidade de dar respostas e os recursos do próprio ou do contexto em que está inserido. Emerge, consequentemente, o conceito de stresse profissional (Ross \& Altmaier, 1994), entendido como a interacção das condições laborais e das características do indivíduo, de tal modo que as exigências que lhe são criadas ultrapassam a sua capacidade de lidar com elas. Mota-Cardoso, Araújo, Ramos, Gonçalves e Ramos (2002), designam estas tensões desenvolvidas no meio profissional por stresse ocupacional.

Alguns estudos com professores portugueses e alunos estagiários (Mota-Cardoso \& cols. 2002; Francisco, Pereira \& Pereira, 2004), revelam também que a actividade docente é geradora de stresse, provocando exaustão emocional, que se torna evidente na desmotivação pessoal e nos elevados índices de absentismo e de abandono. Consideramos significativo evocar, de igual modo, as investigações que destacam a importância de identificar os aspectos positivos (bem-estar) da profissão docente (Jesus, Abreu, Santos \& Pereira, 1992) e não somente os factores negativos (mal-estar). A motivação e a realização do professor, em virtude do conjunto de competências e de estratégias que o indivíduo utiliza para lidar com situações difíceis (coping), são indispensáveis para conseguir fazer face às exigências e dificuldades decorrentes da actividade profissional. Para Bandura (1997), as consequências deste bem-estar no desempenho profissional, sustentado nos sentimentos de optimismo e auto-eficácia, repercutem-se positivamente na aprendizagem e desenvolvimento das crianças.

\section{Estratégias de coping}

O termo coping definido por Lazarus e Folkman (1984) refere-se às estratégias que são utilizadas pelos indivíduos, para lidar com as situações difíceis, de dano, ameaça e desafio. Entre os factores indutores de stresse contam-se aqueles que perturbam o indivíduo ou o sistema em que se insere, desencadeando um estado de tensão e desequilíbrio, exigindo da sua parte mudanças que são essenciais, para que novos estados de equilíbrio sejam alcançados (Boss, 2002). Segundo Lazarus e Folkman (1984), no processo de adaptação a situações de stresse intervém três componentes centrais, nomeadamente os recursos, a percepção da situação e as estratégias de coping. Para Snyder e Dinoff (1999), as estratégias de coping correspondem às respostas emitidas pelos sujeitos, que têm por finalidade diminuir a pressão física, emocional e psicológica ligada aos acontecimentos indutores de stresse. A eficácia das estratégias de coping, segundo estes autores, avalia-se pela capacidade que têm em reduzir de imediato a perturbação sentida, bem como evitar em termos futuros, o prejuízo do bem-estar ou do estado de saúde do ser humano.

Folkman (1984) interpreta coping como os esforços cognitivos e comportamentais utilizados pelo indivíduo, para lidar com situações indutoras de stresse. O coping engloba comportamentos e pensamentos a que o indivíduo recorre para lidar com as situações indutoras de stresse, que podem ser do tipo "problem-focused coping» se estão centradas no problema e «emotion-focused coping», quando estão centradas nas emoções (Folkman \& Lazarus, 1985). Para estes autores, quando um indivíduo avalia uma determinada situação como inalterável utiliza mais o coping orientado para a emoção. Caso uma determinada situação seja avaliada mais como alterável do que inalterável, o indivíduo faz mais uso do coping orientado para o problema. Quando procuramos apoio e conselhos entre os amigos ou familiares, as estratégias de coping de apoio social estão a ser dirigidas para 0 problema, mas se os indivíduos somente necessitam de expressar os seus sentimentos não esperando conselhos, mas sim a compreensão dos outros, então esses esforços estão a ser orientados para um apoio emocional (Pereira, 1991, 1999). Por seu lado Vaz-Serra (2002), considera que, mesmo sendo estas modalidades bem sucedidas, se verifica uma redução do stresse, pois caso contrário a situação mantém-se. Contudo, não há estratégias modelo, pois o nível de eficácia é determinado pelo tipo de recursos que o indivíduo apresenta, assim como pelo tipo de problemas com que se defronta. Neste sentido, o desenvolvimento do processo de coping é influenciado pelas características da personalidade, pela influência cultural e pelos factores situacionais.

Atendendo à necessidade de melhor compreender e lidar com o stresse na profissão docente, pretendemos com este trabalho identificar os mecanismos de coping (estratégias para lidar com situações difíceis) utilizados por 
Educadores de Infância quando se encontram em situações de stresse.

\section{Método}

\section{Participantes}

Neste estudo, a amostra é constituída por 247 Educadores de Infância portugueses, do sexo feminino $(97,2 \%)$ e do sexo masculino $(2,8 \%)$, Licenciados (com estágio pedagógico) e alunos que frequentam o estágio pedagógico. A actividade docente dos Educadores participantes é desenvolvida no sector público $(55,1 \%)$, nas Instituições Particulares de Solidariedade Social IPSS (31,6\%) e no sector privado $(11,1 \%)$.

Os Educadores de Infância que compõem a amostra desenvolvem a sua actividade docente nas seguintes valências: Creche, Pré-Escolar, Actividades de Tempos Livres (ATL), com crianças do zero aos dez anos de idade, com maior incidência no grupo etário dos 2 aos 6 anos de idade (85,3\%). Os Educadores que desenvolvem a sua actividade com grupos etários dos 0 meses aos 2 anos é apenas de $6 \%$, dos 0 meses aos 3 anos é de $1,4 \%$, dos 0 meses aos 5 anos é de $1 \%$ e por último o grupo dos 6 aos 10 anos é de $5 \%$.

A análise descritiva das variáveis idade, tempo de serviço e horário semanal permitiu-nos traçar o seguinte perfil da Educadora de Infância: a média aproximada de idades é de 34 anos $(D P=9,31)$, têm em média 10 anos de tempo de serviço $(D P=9,08)$, em que o valor mínimo e máximo variam entre 0 a 34 anos e trabalham em média 30 horas semanais $(D P=8,95)$. Após recodificação dos valores da idade dos Educadores de Infância em escalões, observamos uma maior frequência no escalão dos 20 aos 30 anos $(41,7 \%)$ e o grupo etário dos 51 aos 57 anos como o menos frequente.

$\mathrm{Na}$ Tabela 1, apresentamos para a amostra total as características sócio-profissionais dos Educadores de Infância. Pela análise da frequência relativa, os dados mostram que o contrato de trabalho é de nomeação definitiva (53\%, efectiva) e $27,1 \%$ enquadram-se noutros tipos de contracto não especificado, estando apenas 19\% em situação de contratadas, ou seja, em situação profissional precária.

Exercem a actividade docente no sector de ensino Público (52,2\%) e nas Instituições Particulares de Solidariedade social - IPSS, $30 \%$ dos inquiridos e apenas $10,5 \%$ exercem a docência no sector privado. Os Educadores são portadores de formação graduada ao nível da licenciatura (70,9\%), seguida do grau de bacharel $(10,5 \%)$. Relativamente à formação pós-graduada apenas $1,6 \%$ possui o grau de mestre. Estes trabalham em média 30 horas semanais e desenvolvem a sua actividade docente com crianças organizadas em grupos verticais $(54,7 \%)$. Estes grupos constituem-se com crianças de diferentes idades, ao contrário dos grupos horizontais, que são constituídos por crianças que nasceram no mesmo ano civil.

Tabela 1. Porcentagem das características Socioprofissionais

\begin{tabular}{|c|c|c|}
\hline \multicolumn{2}{|c|}{ Características Socioprofissionais } & \multirow{2}{*}{$\frac{\text { Porcentagem }}{10,5}$} \\
\hline Grau Acadêmico & Bacharel & \\
\hline & Licenciado & 70,9 \\
\hline & Mestre & 1,6 \\
\hline & Doutorado & \\
\hline & Outro & 4,0 \\
\hline \multirow[t]{3}{*}{ Contracto de trabalho } & Efectiva & 53,0 \\
\hline & Contratada & 19,0 \\
\hline & Outra & 27,1 \\
\hline \multirow[t]{4}{*}{ Sector de ensino } & Público & 52,2 \\
\hline & Privado & 10,5 \\
\hline & IPSS & 30,0 \\
\hline & Outro & 2,0 \\
\hline \multirow[t]{2}{*}{ Tipo de grupo } & Horizontal* & 25,9 \\
\hline & Vertical $^{* *}$ & 54,7 \\
\hline
\end{tabular}

* grupo de crianças com a mesma idade

** grupo de crianças de diferentes idades 


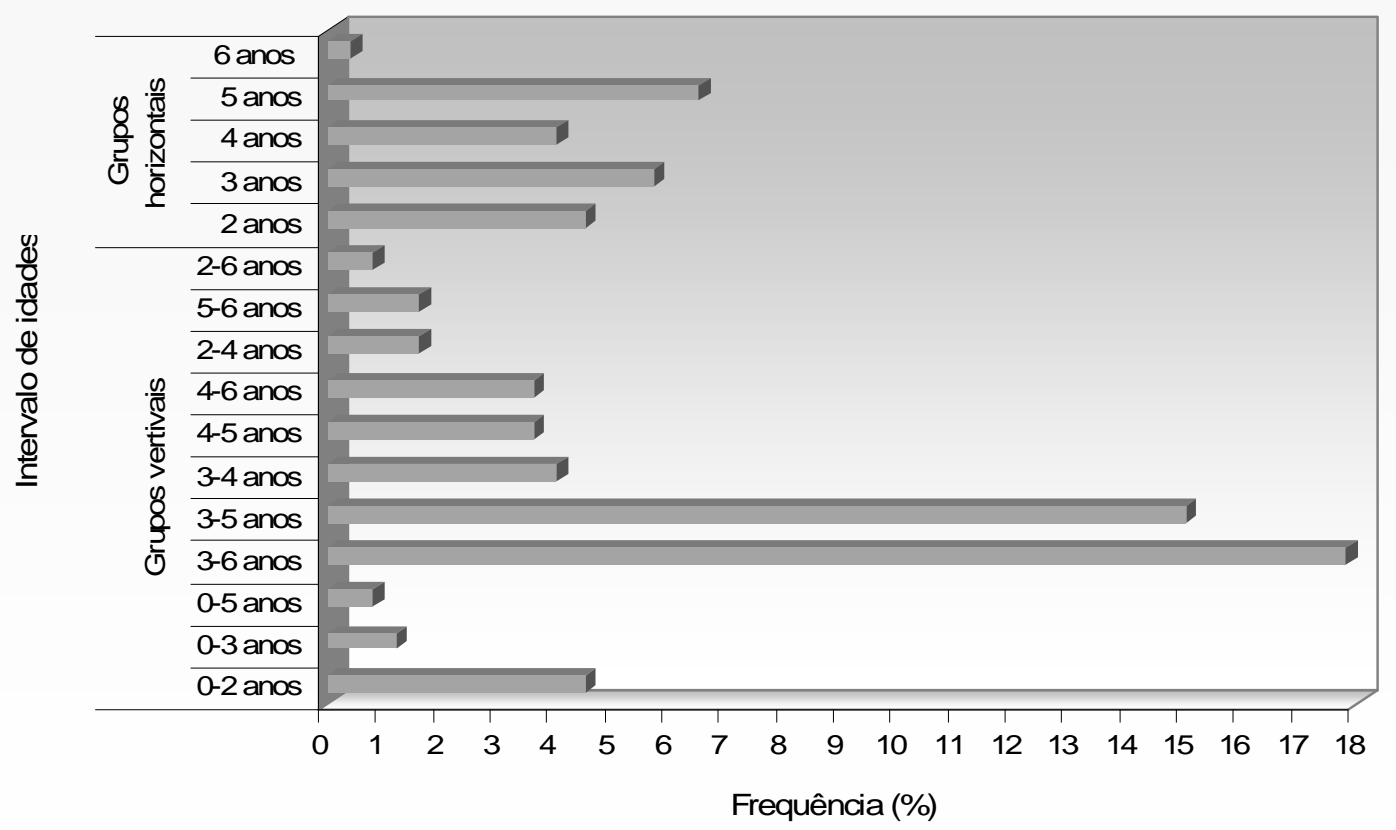

Figura 1. Distribuição dos níveis etários

O gráfico da Figura 1 representa a distribuição dos níveis etários, que fazem parte da estrutura organizacional das turmas, para a amostra em estudo.

$\mathrm{Na}$ valência do Jardim-de-infância a organização dos grupos é elaborada tendo em conta a idade da criança, segundo uma tipologia vertical e horizontal. Neste estudo, os resultados mostram que a tipologia vertical mais frequente agrupa crianças com idades compreendidas entre os 3 e os 6 anos (17,8\%), seguida das idades 3 a 5 anos (15\%). Na tipologia horizontal o nível etário mais frequente é composto por crianças com a idade de 5 anos $(6,5 \%)$, seguida da idade de 3 anos $(5,7 \%)$.

\section{Instrumento de avaliação}

Da bateria Differential Stress Inventory (DSI), de Lefèvre e Kubinger (2004) foi utilizado o questionário Coping, adaptado para a população portuguesa por Gomes, Pereira e Gil (2006), Estratégias de Coping (EC). Este questionário permite avaliar os mecanismos de coping, isto é, como lidariam os indivíduos em situação de stresse profissional. É uma escala tipo Likert composta por 31 itens, em que as respostas são dadas tendo em consideração 4 níveis, em que 1 tem o valor de «quase nunca verdadeiro»; 2 tem 0 valor de "às vezes verdadeiro»; 3 tem 0 valor de «frequentemente verdadeiro" e 4 tem 0 valor de «quase sempre verdadeiro".

Os trabalhos desenvolvidos por Lefèvre e Kubinger (2004), apresentaram no respectivo instrumento psicométrico dois factores: o coping focado na emoção e o coping focado no problema, coincidentes com a revisão da literatura. Atendendo que este estudo é pioneiro em Portugal, optámos por fazer também um estudo psicométrico do referido instrumento, através da avaliação do índice de consistência interna e da análise dos componentes principais (análise factorial).

\section{Procedimentos}

A recolha da amostra decorreu entre Março e Maio de 2006, tendo sido distribuído aos Educadores de Infância o Questionário $E C$, através de um contacto directo nas instituições e por via Internet. Na versão para a Web, procedeu-se a uma readaptação gráfica do questionário, de modo a que este pudesse ser acedido via on-line. Após o seu preenchimento, era enviado pelo próprio para uma base de dados de suporte ao próprio questionário. A divulgação desta modalidade foi feita por convite através de e-mail dirigido a Educadoras de Infância e o acesso era assegurado através de password fornecida pelo próprio investigador, situando a taxa de retorno em $37,9 \%$, para as respectivas modalidades. As questões éticas foram respeitadas, a participação foi voluntária e cada questionário era acompanhado de carta explicativa dos objectivos, das condições da pesquisa e da confidencialidade dos dados. Para a análise de dados utilizámos 0 programa estatístico SPSS (Statistical Package of Social Science), versão 14,0 para Windows, tendo sido feitas estatísticas descritivas e inferenciais. 


\section{Resultados}

Participaram neste estudo 247 Educadores de Infância, em que $85,8 \%$ são Educadores diplomados (com estágio) e $14,2 \%$ são alunos da Licenciatura em Educação de Infância, que se encontram no ano de estágio pedagógico, não tendo ainda terminado o estágio (sem estágio), em que $97,2 \%$ são do sexo feminino e $2,8 \%$ do sexo masculino, variando a idade entre 20 e 57 anos $(M=33,85 ; D P=9,31)$.

$\mathrm{Na}$ análise descritiva do instrumento $E C$, para as duas dimensões que caracterizam as estratégias de coping, observamos que os itens que apresentam valores médios mais altos nas estratégias de coping focadas na emoção são: quando estou sob stresse esforço-me por voltar a encontrar o meu próprio ritmo $(M=3,20 ; D P=0,71)$, esforço-me por me controlar $(M=3,09 ; D P=0,79)$, tento fazer algo em relação ao meu estado de tensão $(M=2,95$; $D P=0,86)$ e penso em algo agradável $(M=2,69 ; D P=$ $0,94)$. Já as estratégias de coping focadas no problema são: quando estou sob stresse falo da minha situação a alguém $(M=2,68 ; D P=0,95)$, peço conselho a outras pessoas $(M=2,52 ; D P=0,94)$, peço ajuda a outras pessoas $(M=2,41 ; D P=0,90)$ e tento passar algum do trabalho para outras pessoas $(M=1,83 ; D P=0,82)$.

Efectuámos a análise de componentes principais (ACP), rotação tipo varimax e para os valores próprios superiores a 1 (regras do eigenvalue), extraímos 2 factores. Tendo em consideração os objectivos a que nos propusemos, nomeadamente traduzir e adaptar para Portugal e para a língua portuguesa, decidimos excluir onze itens, por apresentarem valores de saturação inferiores a .350 (factor loadings). O primeiro factor (Factor1), que intitulámos "coping focalizado na emoção" engloba 13 itens $(1,4,5,8,18,19,20,21,22,24,26,29$, 31) e explica $17,57 \%$ da variância. O segundo factor (Factor2), que intitulámos «coping focalizado no problema» engloba 7 itens $(6,7,9,10,23,27,28)$, explicando $9,24 \%$ da variância (Tabela 2). Os dois factores explicaram $26,81 \%$ da variância total.

Tabela 2. Análise dos Componentes Principais (ACP)

\begin{tabular}{|c|c|c|c|}
\hline \multirow{2}{*}{ Itens } & \multirow{2}{*}{$\begin{array}{l}\text { Descrição } \\
\text { Quando estou sob estresse... }\end{array}$} & \multirow{2}{*}{$\begin{array}{l}\text { Factor1 } \\
\text { Emoção }\end{array}$} & \multirow{2}{*}{$\begin{array}{c}\text { Factor } 2 \\
\text { Problema }\end{array}$} \\
\hline & & & \\
\hline 1 & ...esforço-me por voltar a encontrar o meu próprio ritmo. & 0,532 & \\
\hline 4 & ...tento estabelecer prioridades. & 0,580 & \\
\hline 5 & ...penso na forma como lidei com situações semelhantes no passado. & 0,514 & \\
\hline 8 & ...organizo o meu ambiente da melhor forma possível. & 0,653 & \\
\hline 18 & ...digo a mim mesmo palavras encorajadoras. & 0,472 & \\
\hline 19 & ...esforço-me por me controlar. & 0,657 & \\
\hline 20 & ...tento distrair-me. & 0,625 & \\
\hline 21 & ...traço um plano. & 0,631 & \\
\hline 22 & ...penso em algo agradável. & 0,705 & \\
\hline 24 & ...penso em como poderia tornar as coisas mais fáceis para mim. & 0,497 & \\
\hline 26 & ...penso naquilo que terá causado esse stresse. & 0,563 & \\
\hline 29 & ...tento acalmar-me. & 0,646 & \\
\hline 31 & ...tento fazer algo em relação ao meu estado de tensão. & 0,680 & \\
\hline 6 & ...peço ajuda a outras pessoas. & & 0,646 \\
\hline 7 & ...tento passar algum do trabalho para outras pessoas. & & 0,357 \\
\hline 9 & ...falo da minha situação a alguém. & & 0,552 \\
\hline 10 & ...penso naquilo que se vai passar a seguir na minha cabeça. & & 0,611 \\
\hline 23 & ...paro de fazer tudo. & & 0,483 \\
\hline 27 & ...peço conselho a outras pessoas. & & 0,597 \\
\hline 28 & ...digo a mim mesmo que as outras pessoas também estão sob stresse. & & 0,515 \\
\hline \multicolumn{2}{|c|}{ \% Variância explicada } & 17,57 & 9,24 \\
\hline \multicolumn{2}{|c|}{$\alpha$ dos Factores } & 0,85 & 0,71 \\
\hline
\end{tabular}


Tabela 3. Médias e Desvios-Padrão dos Factores das Estratégias de Coping

\begin{tabular}{lccc}
\hline Factores & $\mathrm{N}$ & $\mathrm{M}$ & $\mathrm{DP}$ \\
\hline F1 emoção & 235 & 36,56 & 6,78 \\
F2 problema & 233 & 15,12 & 3,70 \\
\hline
\end{tabular}

Atendendo à depuração da escala, obtivemos para a versão reduzida um Alfa de 0,83 (20 itens) considerado muito adequado e indicador de uma boa consistência interna deste instrumento. Determinámos ainda o Alfa de Cronbach para os respectivos factores, sendo o valor Alfa de 0,85 para o Factor1 (13 itens) e de 0, 71 para o Factor2 (7 itens), valores estes considerados adequados e indicadores de boa consistência interna dos respectivos factores.

Procedemos também à comparação dos resultados do presente estudo com as versões originais da língua inglesa e portuguesa, quer a nível dos factores identificados (emoção e problema), quer a nível dos valores globais de Alfa. Assim a análise factorial dos componentes principais e dos níveis de consistência interna por nós realizada, foi semelhante e coincidente com as versões originais. Os estudos em língua inglesa de Lefèvre e Kubinger (2004), apresentaram um Alfa global de 0,73 e nos primeiros estudos da versão portuguesa de Gomes, Pereira e Gil (2006), um Alfa global de 0,77.

Na Tabela 3, apresentamos as médias e desvio-padrão obtidos no instrumento Estratégias de Coping, para a amostra total. Pela análise descritiva dos factores, identificámos que os Educadores utilizam mais as estratégias de coping focadas na emoção, Factor1 $(M=$ $36,56 \% ; D P=6,78$ ) do que focadas no problema Factor2 $(M=15,12 \% ; D P=3,70)$.

Contudo, quando comparados os Educadores com estágio e sem estágio, relativamente à utilização do tipo de estratégias de coping que usam, os resultados obtidos com o teste T-student indicam não haver diferenças estatisticamente significativas, quer ao nível das estratégias de coping focadas na emoção $(t=-1,945$; $g l=233 ; p=0,053)$, quer ao nível das estratégias de coping focalizado no problema $(t=-1,445 ; \quad g l=231$; $p=0,150)$.

\section{Discussão}

Os resultados deste estudo exploratório foram de encontro aos objectivos propostos, especificamente na identificação das estratégias de coping e no estudo das características do instrumento. Relativamente ao instrumento de avaliação, este apresentou uma estrutura de dois factores (coping na emoção e coping no problema) e uma boa consistência interna, corroborando assim os estudos já realizados com este instrumento por outros investigadores (Lefèvre \& Kubinger, 2004; Gomes, Pereira \& Gil, 2006).

Os Educadores de Infância licenciados e em estágio, perante situações indutoras de stresse utilizam mais as estratégias de coping focadas na emoção, do que as focadas no problema. Essas estratégias são do tipo: quando estou sob stresse esforço-me por voltar a encontrar o meu próprio ritmo, por me controlar, acalmarme, fazer algo em relação ao seu estado de tensão e estabelecer prioridades e pensar na causa que desencadeou 0 stresse. Partilhar, pedir ajuda ou aconselhar-se com alguém são categorias de coping do tipo apoio social. Contudo, quando comparadas as estratégias de coping focadas na emoção com as estratégias de coping focadas no problema, os indivíduos recorrem mais às primeiras. $O$ desenvolvimento de práticas educativas com grupos de crianças de diferentes idades (grupos verticais) e, por conseguinte, diferentes fases do desenvolvimento bio-psico-social, confere ao Educador de Infância uma especificidade docente, que poderá potenciar uma maior exposição a situações indutoras de stresse, recorrendo portanto à utilização de estratégias de coping focadas na emoção (Gomes, 2006).

No que concerne aos tipos de estratégias utilizadas, Folkman e Lazarus (1985) referem que estas são influenciadas pelos contextos, pelas experiências passadas e pelas características de personalidade. Estes argumentos poderão explicar as diferentes estratégias dos Educadores de Infância, que com o tempo aprendem a lidar mais facilmente com os problemas. Dependendo das situações, as estratégias focalizadas na emoção podem aliviar a tensão numa fase inicial, possibilitando uma maior adaptação aos contextos, facilitando assim a tomada de decisões para a resolução dos problemas e evitar situações de doença (Vaz-Serra, 2002; Pereira, 2006). Também Gomes e cols. (2006), mostram que os Educadores em estágio e com estágio utilizam diferentes estratégias de coping, sendo que a prática profissional é uma variável a ser considerada.

A Prática Pedagógica dos Educadores de Infância (realizada no ano de estágio), não indicou ser este momento indutor de stresse. Contudo, a investigação nesta área tem vindo a demonstrar que o ano de estágio é particularmente indutor de stresse, devendo para o efeito serem encontradas estratégias de combate ao stresse no ano de estágio, como tem vindo a ser realçado por Francisco e cols. (2004). Estes dados permitem-nos alertar para a necessidade de se intervir não só na formação 
inicial, mas também na formação contínua dos Educadores de Infância, especificamente ao nível da promoção do desenvolvimento pessoal e profissional.

Atendendo a que este estudo é exploratório, os resultados deverão ser lidos com alguma precaução, devido ao tipo de instrumentos utilizados bem como à não representatividade da amostra. Além disso, tendo em consideração de que é o primeiro estudo realizado em Portugal com esta população e instrumento de avaliação, não temos dados que nos permitam comparar. Por se tratar de investigação com indivíduos e comportamentos humanos, não foram controladas as variáveis parasitas, inerentes a este tipo de estudo.

São necessárias mais investigações que nos permitam aprofundar esta temática, nomeadamente replicar estudos para validação do instrumento a nível nacional, bem como realizar estudos longitudinais para averiguar da variabilidade das estratégias de coping, ao longo da prática profissional.

\section{Referências}

Abrantes, N. (2006). Parcerias na Construção Curriculares em Educação de Infância: os Saberes das Crianças em Diálogo com a Intervenção do Educador. Em J. Tavares, A. M. S. Pereira, C. Fernandes \& S. Monteiro (Orgs.), Actas do Simpósio Internacional de Activação do Desenvolvimento Psicológico (pp. 300-307). Aveiro: Universidade de Aveiro.

Bandura, A. (1997). Self Efficacy. The Exercise of Control. Newyork: NJ Freeman.

Boss, P. (2002). Family Stress Management. A Contextual Approach. California: Sage Publications.

Folkman, S. (1984). Personal Control and Stress and Coping Processes. A Theoretical Analysis. Journal of Personality and Social Psychology, 46(4), 939-952.

Folkman, S., \& Lazarus, R. S. (1985). If it Changes it Must be Aprocess: A Study of Emotion and Coping During Three Stages of a College Examination. Journal of Personality and Social Psychology, 48, 150-170.

Francisco, C., Pereira, A., \& Pereira, G. (2004). Fontes de Stresse do Estudante Estagiário: Contributos da Supervisão para o Bemestar do Aluno. Em J. Ribeiro \& I. Leal (Eds.). Actas do 5 Congresso de Psicologia da Saúde, (pp. 111-117) Lisboa: Fundação Calouste. ISPA Edições.
Gomes, R. (2006). O Stresse na Infância e o Impacto das Actividades de Iniciação às Ciências Naturais. Dissertação de Mestrado, Universidade de Aveiro, Aveiro-Portugal.

Gomes, R., Pereira, A., \& Gil, V. (2006). Stresse e Coping nos Educadores de Infância. Em J. Tavares, A. Pereira, C. Fernandes \& S. Monteiro (Org.), Actas do Simpósio Internacional de Activação do Desenvolvimento Psicológico (pp. 84-89). Aveiro: Universidade de Aveiro.

Guerra, M. (2002). A Escola que Aprende. Lisboa: CRIAP, ASA.

Jesus, S., Abreu, M., Santos, E., \& Pereira, A. (1992). Estudo dos Factores de Mal-estar na Profissão Docente. Psychologica, 8, 5160.

Lazarus, R., \& Folkman, S. (1984). Stress, Appraisal and Coping. New York: Springer.

Lefèvre, S., \& Kubinger, K. D. (2004). Differential Stress Inventory. Differentielles Stress Inventar (DSI). Test: Software und Manual. Mödling: Wiener Testsystem/ Schuhfried

Leite, C. (2002). Para uma Escola Curricularmente Inteligente. Lisboa: Edições ASA.

Mota-Cardoso, R., Araújo, A., Ramos, R., Gonçalves, G., \& Ramos, M. (2002). O Stress nos Professores Portugueses Estudo IPSSO 2000. Porto: Porto Editora.

Pereira, A. (1991). Coping, Auto-conceito e Ansiedade Social: Sua Relação com o Rendimento Escolar. Dissertação de Mestrado, Universidade de Coimbra, Coimbra-Portugal.

Pereira, A. (1999). Resiliência e Estratégias de Coping no Encontro do Terceiro Milénio. Em IV Congresso da Sociedade Portuguesa de Ciências da Educação (Org.), Investigar e Formar em Educação (Vol. 1, pp 373-378). Aveiro.

Pereira, A. (2006). Stresse e Doenças: Contributos da Psicologia da Saúde na Última Década. Em Isabel Leal (Coord.), Perspectivas em Psicologia da Saúde (pp. 145-167). Coimbra: Quarteto Editora.

Ross, R., \& Altmaier, E. (1994). Intervention in Occupational Stress. London, Thousand Oaks \& New Delhi: Sage Publications.

Snyder, C., \& Dinoff, B. (1999). Coping: Where Have You Been? Coping - The Psychology of What Works. Oxford: Oxford University Press.

Vaz-Serra, A. (2002). O Stress na Vida de Todos os Dias. Coimbra: Gráfica de Coimbra, Ltda.

Recebido em: 25/01/2008

Revisado em: 10/11/2008

Aprovado em: 17/12/2008 


\section{Sobre as autoras}

Rosa Maria Silva Gomes (rosa.gomes@ua.pt) é Mestre em Comunicação e Educação em Ciência pela Universidade de Aveiro. Técnica Superior no Círculo de Animação e Investigação Pedagógica para a Infância do Departamento de Ciências da Educação da Universidade de Aveiro.

Anabela Maria Sousa Pereira (anabelapereira@ua.pt) é Doutorada em Psicologia e Professora no Departamento de Ciências da Educação da Universidade de Aveiro.

\section{Endereço para correspondência}

Rosa Maria Silva Gomes

Departamento de Ciências da Educação

Universidade de Aveiro

Campus Universitário de Santiago

3810-193 Aveiro

\section{Origem do trabalho}

Gomes, R. M. (2006). O stresse na infância e o impacto das actividades de iniciação às ciências naturais. Dissertação de Mestrado. Aveiro: Universidade de Aveiro. 